\title{
Control Indicators for Resource Leveling in Project Network Planning
}

\author{
Mengrui Shao ${ }^{1, a}$, Xun Liu'b \\ 1School of JiangNan University, Wuxi 214122, China. \\ 1School of JiangNan University, Wuxi 214122, China. \\ aMengrui Shao@qq.com
}

\begin{abstract}
Keywords: project network planning; resource leveling; construction management; control indicators
\end{abstract}

\begin{abstract}
In construction project management, the increase of project cost due to resource fluctuation is widespread. This paper proposed new resource leveling control indicators reflecting resources idle and resources mobilizing. According to the disadvantages of traditional control indicators of resource leveling in project network planning, considering the substantial increased costs due to resources fluctuation, based on the classification of extra costs, this study found out the relationship between resource imbalance and extra costs, analyzed resources idle and resources mobilizing cost variation caused by resource imbalance. Thus, the control indicators of resources and resources mobilizing reflecting resource leveling are proposed. In addition, based on case study, the feasibility of the control indicators proposed in this paper for resource leveling in project network planning was validated. The information is valuable in practical application for the resource leveling optimization in engineering project management.
\end{abstract}

\section{Introduction}

The practical significance of resource leveling optimization is widely known. Resource leveling mainly considers the balance of the resource usage over time in the course of project implementation in order to avoid resource supply bottlenecks and affecting the normal operation of the project. The main problem to be solved by the research on resource leveling optimization in engineering construction is describing the resource balance validly, and that is to design rational and effective evaluation indicators of resource leveling. In the study of indicators for resource leveling in project network planning, aimed at the resource balance problem,Bandellonietal(1994)[1], Hegazy(1999)[2],Son and Skibniewski(1999)[3] used the ratio of maximum resource consumption to the intensity of resource consumption in the construction process which is also called imbalance coefficient to solve resource leveling problem in project. They used algorithms such as dynamic programming, the genetic algorithm and heuristic algorithm to solve resource leveling problem in project. Easa(1989) $)^{[4]}$, Senouci and Adeli(2001) ${ }^{[5]}$ and Senouci and Eldin $(2004)^{[6]}$ applied integer linear programming, neural dynamics and the genetic algorithm to solving the problem of resource leveling by using the minimum absolute value between resource requirements and average resources as the objective function which is also called the largest absolute deviation. The literature [7-15] used variance or standard deviation as evaluation indicators for resource leveling and used the genetic algorithm, integer linear programming, particle swarm optimization algorithm, simulated annealing and so on to solve the problem of resource leveling in project and form rich ideas and methods about resource leveling optimization. Chenxingan $(1993)^{[16]}$ and others suggested application rules to solve the problems by using the basic principles of difference method, and gave calculation formulas. The literature [17-19] used the difference as the evaluation indicator for resource leveling and used genetic algorithm and linear programming to solve formulas. Rich achievements have been made either in the thinking of resource leveling optimization, also in method of equilibrium optimization when using unbalance coefficient, mean absolute deviation, range, variance or standard deviation as the evaluation indicator for resource leveling. But from that resource leveling is the ultimate goal of reducing increased costs deriving from resource 
fluctuations, these indicators do not reflect the substance that increased costs have reduced due to reducing resources fluctuation in project network planning. That is, these indicators are not using the opportunity cost which is the indicator of cost to define. Aiming at this problem, this paper, proposed metrics for resource consumption which causes changes of cost from the angle resources idle and resources mobilizing cost variation, and made these as evaluation indicators for resource leveling in project network.

\section{Traditional Evaluation Indicators for Resource Leveling and Disadvantages}

\section{1Traditional Evaluation Indicators for Resource Leveling}

At present, common evaluation indicators for resource leveling contain the following four kinds: unbalanced factor、variance $\sigma^{2} 、$ maximum absolute deviation $\Delta R$ and range $\Delta R_{\max \text { min }}$.

(1) Unbalanced Factor V

Unbalanced factor is used to evaluate the relationship between the maximum intensity of resource consumption and average resource consumption intensity. Unbalanced factor can react directly the impact proportion of the maximum resource consumption on the entire resource consumption curve.

$$
V=\frac{R_{\max }}{R_{M}}
$$

In equation (1), $R_{\max }$ represents the maximum value of the resource requirements at all times, $V$ represents the unbalanced factor of resource requirements, $R_{M}$ represents the average value of the resource requirements at all times and they can be calculated as follows:

$$
R_{M}=\frac{1}{T} \sum_{t=1}^{T} r_{t}
$$

In equation (2) $T$ represents the duration of the project and $r_{t}$ represents the resource consumption quantity on the $t$ th day of the period. When the unbalanced factor is used as the evaluation indicator, the smaller of unbalanced factor $V$ the better balance of resource intensity of the instruction project.

(2) Maximum Absolute Deviation $\Delta R$

Maximum absolute deviation $\Delta R$ means comparing the number of daily consumption of resources with the average resource requirements of all time. Maximum absolute deviation can be calculated as equation (3):

$$
\Delta R=\max \{|R(t)-\bar{R}|\}
$$

In equation (3), $R(t)$ represents the daily demands for resources. The smaller of $\Delta R$ the better balance of resource intensity.

(3) Range $\Delta R_{\max -\min } \quad \Delta R_{\max -\min }=\Delta R_{\max }-\Delta R_{\min }$

In the deviation, $\Delta R_{\max }$ represents the the maximum value of resource intensity in project while $\Delta R_{\min }$ represents the minimum value.

(4) Variance $\sigma^{2}$

Calculation formula of variance $\sigma^{2}$ :

$$
\sigma^{2}=\frac{1}{T} \int_{0}^{T}[R(t)-\bar{R}]^{2} d t
$$

It uses the notion of sample variance in statistics to measure the balance of the amount of resources needed per unit of time. Considering that objects of statistics are all discrete resource requirements in practical engineering, so variance $\sigma^{2}$ is normally calculated as the following form: 


$$
\sigma^{2}=\frac{1}{T} \sum_{t=1}^{T}[R(t)-\bar{R}]^{2}
$$

In equation (6), $T$ represents the total duration of a project, $R(t)$ represents the intensity of resource consumption on the $\mathrm{t}$ th day of the period, $\bar{R}$ represents the average resource consumption intensity in construction and the smaller of $\bar{R}$ the better balance of resource intensity.

\section{2The Disadvantages of Traditional Evaluation Indicators for Resource Leveling}

In the traditional four types of evaluation indicators for resource leveling, unbalanced factor ignored the possibility that resource consumption is lower than the negative values of the average intensity of resources and has the maximum absolute value. What's more, they can't react the details of the process of resource consumption curve. Variance (SD) indicators are now widely used by scholars in the resource leveling optimization and they become the most important indicators to describe the resource leveling. Variance ( SD ) indicators can describe the characteristics of resource consumption intensity well and are applied in tasks such as evaluation of resource leveling and resource leveling horizontal comparison and so on. With the deepening of research on resource leveling optimization, the author thinks it is necessary to classify extra costs arising from resource imbalance and find out the relationship between resource imbalance and extra costs to grasp the focus of resource leveling optimization and improve the effectiveness and efficiency of optimization. In all kinds of project network planning, the rise and fall of resource consumption curve contributes to the intensity of resource consumption rising and falling. Several peak values in the curve generated due to the difference of resource consumption in different construction periods. For the construction periods between two adjacent peek values, theoretically, a construction enterprise can make the following two options: One is to have the extra resources between adjacent peaks in the idle state while using them in the next peak what causes resources idle cost. Another option is to enable the flow of resources, that is, to withdraw resources from the construction site, and enter construction sites engaging in productive work again when the next peak comes what causes the construction resource mobilizing cost. Therefore, the extra costs arising from resource imbalance are classified as:(1) construction resource idle

Through the classification of the extra costs arising from resource imbalance, we can see the ultimate goal of resource leveling optimization is to decrease the construction resources idle and mobilizing cost caused by resource imbalance. The essence of the resource leveling optimization is to decrease the cost of construction resources idle and resources mobilizing. This is the shortcomings of traditional evaluation indicators of resource leveling such as unbalanced factor, maximum absolute deviation, range and variance.

\section{Evaluation Indicators of Resource Consumption causing the changes of Resource Costs}

Through the above classifications of extra costs caused by the resource imbalance, it can be seen that the essence of the resource leveling optimization is to decrease the cost of construction resources idle and resources mobilizing. Designing a reasonable and effective evaluation indicator of resource leveling to assess the number of resources idle and resources mobilizing in the process of engineering construction is very important.

\section{1Measurement of Resource Consumption causing the changes of Resources Idle Cost}

In the process of construction, the quantity of resources idle needs to be as little as possible. Measurement of the number of resources idle of each day within the engineering construction period is demanded. The number of resources idle on the $\mathrm{t}$ th day of the period is $R I_{t}$, then:

$$
R I_{t}=\operatorname{Min}\left\{\operatorname{Max}\left(r_{1}, r_{2}, \cdots, r_{t}\right), \operatorname{Max}\left(r_{t}, r_{t+1}, \cdots, r_{T}\right)\right\}-r_{t}
$$

In the equation above, $r_{t}$ represents the demand for resources on the $\mathrm{t}$ th day of the period and $T$ represents the total duration of a project. When summarizing the number of resources idle each day in construction period, the total number of resources idle in the project construction period is as formula (8) below: 


$$
R I=\sum^{T}\left[\operatorname{Min}\left\{\operatorname{Max}\left(r_{1}, r_{2}, \cdots, r_{t}\right), \operatorname{Max}\left(r_{t}, r_{t+1}, \cdots, r_{T}\right)\right\}-r_{t}\right]
$$

\section{2Méasurement of Resource Consumption causing the changes of Resources Mobilizing Cost}

In realistic problems, engineering project managers mostly use the peak value of resources and the difference of resources between the adjacent units of time to evaluate the balance of use of the project's resources. Peak value of resources is directly associated with the resource supply and inventory capabilities. Managers are keen to the lowest supplies in the market around the construction projects. If the number exceeds the supply, the cost of resource supply will be increased and this is what managers do not want to see, but also the scientific requirements of the project's own benefit. The smaller the difference of resource utilization between adjacent units, the more convenient supply of resources and the smaller flow of resources and the bigger elasticity of resource usage. What's more, the resource coordination capacity of project resource itself can be ensured and this is also favored by managers in site. Therefore, in the course of the implementation of construction projects, the number of secondary flow of construction resource is demanded to be as less as possible to improve the resource coordination ability of project resource itself. The ideal resource configuration is gradually increasing the resources in the resource entry stage to reach the peak of construction resources while decreasing the resources gradually in the resource exits stage. So, it is needed to firstly evaluate the total number of resource fluctuations RF throughout the entire construction period, as formula (9) below:

$$
R F=\left[r_{1}+\sum_{t=1}^{T-1}\left|r_{t}-r_{t+1}\right|+r_{T}\right]
$$

In the equation above, $r_{t}$ represents the resource requirements on the $t$ th day of the period and $T$ represents the total duration of a project. So, the total daily increasing number of resources $T$ is half of the number of resource fluctuations $R F$ in construction period $T$, that is :

$$
H=\frac{1}{2} R F
$$

Equation (10) contains the maximum peak value of resource requirements $R_{\max }$, so this part should be subtracted when measuring the resource leveling degree in the amount of resources mobilizing, then the amount of resources mobilizing can be calculated as the formula (11)below, that is:

$$
R M=\frac{1}{2} R F-R_{\max }=\frac{1}{2}\left[r_{1}+\sum_{t=1}^{T-1}\left|r_{t}-r_{t+1}\right|+r_{T}\right]-R_{\max }
$$

In the equation above, $R_{\max }$ is the peak value of resource consumption, that is $R_{\max }=\operatorname{Max}\left(r_{1}, r_{2}, \cdots, r_{T}\right)$.

\section{Resource Leveling Optimization Model}

Fixed Duration---Resource leveling optimization aims to optimize the resources in accordance with the distribution of time in line with targets through changing the start and finish times of work. The main ways to optimize are:( ) take advantage of jet lag and postpone the start time of certain operation $\odot$ change the working time for certain jobs or make some temporary disruption of operations at the time of resource constraints when conditions permit to reduce the imbalance of resource use. In the modeling process, this paper optimized mainly by adjusting the start time for the operation in progress network and temporary disruption of process at the time of resource constraints is temporarily not allowed and its prerequisites are following:( The relationship such as who is the former and who is the later of the process activities is known and fixed and the later work can't be done until the former work is finished@ In the progress of network optimization process, the duration of each process remains unchanged. (3) Work interruptions are not allowed and 
the continuity of process in the progress of network should be ensured. (4) Resource requirements of each process should be maintained at a constant level during the whole project implementation what means that we can't add or subtract a resource temporarily. (5) Resource requirements of each process is fixed and known. (6) Assuming that the lead time is included in the working procedure duration. (7) Without considering the engineering cost and the problems of crash. The goal of resource leveling optimization is to look for scheduled start time for each activity and make the required resource consumption quantity which causes the changes of resource is the minimum value. The optimization model can be described in equation (12):

$$
\begin{aligned}
& \min F=\min R I \quad \text { or } \min R M \\
& \text { s.t. }\left\{\begin{array}{l}
E S_{i} \leq S_{i} \leq L S_{i}, i=1,2, \cdots, N \\
S_{i}+D_{i} \leq S_{j}, j \in \operatorname{Succ}(i)
\end{array}\right.
\end{aligned}
$$

In the equation above, $S_{i}$ represents the scheduled start time of activity $i$ and $S_{j}$ represents the scheduled start time of activity $j . E S_{i}$ is the earliest start time of activity $i$ and $L S_{i}$ represents the latest start time of activity i. $D_{i}$ represents the duration of activity $i, N$ represents the number of engineering activities. $\operatorname{Succ}(i)$ is the activity after activity $i$. Among these, $R I$ can be calculated by equation (8) and $R M$ can be calculated by equation ( 11 ).

\section{Conclusions}

Resource leveling problem has always been issues of concern to project managers and researchers, but most of the relevant researches focused on the innovation of optimization and ignored the innovation of problems. Especially in recent years, emergence of some intelligent bionic algorithm has attracted many researchers to use it in solving resource leveling problem. While many researchers have lacked thinking about the nature of resource leveling problem. Based on the new awareness of extra costs of resources caused by resource imbalance, this paper pointed out that the essence of resource leveling is reducing the unintended opportunity cost of resources totally. Moreover, this paper analyzed the cost of resources idle and resources mobilizing caused by resource imbalance and then essentially proposed evaluation indicators reacting the number of resources idle and resources mobilizing which measuring the degree of resource leveling. On this basis, it is possible to carry out resource leveling optimization more scientifically and reasonably to improve control of resources and economic benefits of the project. While the relationship between resource imbalance and extra costs need further studies.

\section{References}

[1]Bandelloni, M., M. Tucci, and R. Rinaldi.Optimal Resource Leveling Using Non-Serial Dynamic-Programming[J]. European Journal of Operational Research, 1994. 78(2): 162-177.

[2]Hegazy, T., Optimization of resource allocation and leveling using genetic algorithms[J]. Journal of Construction Engineering and Management, 1999. 125(3): 167-175.

[3]Son, J. and M.J. Skibniewski. Multiheuristic approach for resource leveling problem in construction engineering: Hybrid approach[J]. Journal of Construction Engineering and Management, 1999. 125(1): 23-31.

[4]Easa, S.M., Resource Leveling in Construction by Optimization[J]. Journal of Construction Engineering and Management, 1989. 115(2): 302-316.

[5]Senouci, A.B. and H. Adeli. Resource scheduling using neural dynamics model of Adeli and Park[J]. Journal of Construction Engineering and Management, 2001. 127(1): 28-34.

[6]Senouci, A.B. and N.N. Eldin. Use of genetic algorithms in resource scheduling of construction projects[J]. Journal of Construction Engineering and Management, 2004. 130(6): 869-877. 
[7]Chan, W.T., D.K.H. Chua, and G. Kannan. Construction resource scheduling with genetic algorithms[J]. Journal of Construction Engineering and Management, 1996. 122(2): 125-132.

[8]Mattila, K.G. and D.M. Abraham. Resource leveling of linear schedules using integer linear programming[J]. Journal of Construction Engineering and Management, 1998. 124(3): 232-244.

[9]ChenZhiyong,DuZhida,ZhouHua.ResearchontheunlimitedresourcelevelingoptimizationwithPSO[ J]. Journal of Civil Engineering, 2007(02): 93-96.[In Chinese].

[10] Li Xingmei, Qi Jianxun, Su Zhixiong.ResearchonResourceLevelingBased on theAnalysis of Activity floats[J]. Management science in China, 2007(01): 47-54.[In Chinese].

[11] Qi Jianxun,WangQiang,Jia Haihong. new method of research on resource leveling Optimization Based on entropy weight and particle swarm[J]. Management science in China, 2008(01): 90-95.[In Chinese].

[12]Pang Nansheng,Ji Cangming. Active multi - mode and Discrete resource leveling optimization Under the Generalized sequential [J]. Journal of Systems Engineering, 2011(04): 538-545.[In Chinese].

[13]Rieck, J., J. Zimmermann, and T. Gather. Mixed-integer linear programming for resource leveling problems[J]. European Journal of Operational Research, 2012. 221(1): 27-37.

[14]Ponz-Tienda, J.L., et al., The Resource Leveling Problem with multiple resources using an adaptive genetic algorithm[J]. Automation in Construction, 2013. 29: 161-172.

[15]OuYang hongxiang,LiuBingsheng,LiXin. Genetic Algorithm for Multi - resource leveling Optimization of network planning [J]. Journal of Wuhan University of Technology (Information and Management Engineering Edition), 2013(02): 180-182+190.[In Chinese].

[16]ChenXingan,LiangShaozhou. Duration of network plan - Resource Optimization Method [J]. Operations research and management, 1993(Z1): 29-36.[In Chinese].

[17]Chua, D.K.H., W.T. Chan, and G. Kannan. Scheduling with co-evolving resource availability profiles[J]. Civil Engineering Systems, 1996. 13(4): 311-329.

[18]Leu, S.S. and C.H. Yang. GA-based multicriteria optimal model for construction scheduling[J]. Journal of Construction Engineering and Management, 1999. 125(6): 420-427.

[19]Son, J. and K.G. Mattila. Binary resource leveling model: Activity splitting allowed[J]. Journal of Constuction Engineering and Management, 2004. 130(6): 887-894. 